\title{
Predictability of Outcome in Laparoscopic Gastric Banding
}

\author{
Andreas Thalheimer* Marco Bueter* Alexander Wierlemann Caroline Lager \\ Christian Jurowich Christoph-Thomas Germer Martin Fein
}

Department of General Surgery, University of Wuerzburg Hospital, Wuerzburg, Germany

\section{Key Words}

Gastric banding - Morbid obesity - Outcome predictors · Preoperative selection

\section{Summary}

Background: The success rate of laparoscopic adjustable gastric banding (LAGB) in the treatment of morbid obesity is very variable. A reasonable preoperative selection of eligible patients seems to be important for a successful outcome of LAGB. In the present study, criteria were investigated to predict the outcome of LAGB. Methods: 85 morbidly obese patients were operated with LAGB between 1999 and 2005. 71 of these patients were analysed according to several possible predictive parameters of success or failure of LAGB. Success was defined as excess body weight loss (EBWL) $>50 \%$ without band removal, failure was defined as EBWL $<20 \%$ and/or band removal. Median follow-up was 27 months (range 8-90 months). Results: After LAGB a median EBWL of $43 \%$ (-41 to $171.5 \%)$ was observed in all patients with a decrease in BMI of $8.0 \mathrm{~kg} / \mathrm{m}^{2}\left(-9\right.$ to $\left.35 \mathrm{~kg} / \mathrm{m}^{2}\right)$. The success rate after LAGB was $37 \%$, the failure rate $19.7 \%$. Female sex $(p=0.023)$, baseline weight $(p=0.024)$, and eating behaviour after LAGB ( $p=0.008$ ) were significant predictors of success following LAGB, whereas complications such as port dislocation and reoperation after LAGB did not have a significant impact on a successful course following LAGB. Significant predictors of failure were male sex $(p=0.038)$ and missing physical activity after LAGB $(p=0.045)$, whereas the eating behaviour did not have a significant effect concerning failure following LAGB. Baseline excess body weight (EBW) was identified as an independent predictor of failure in a multivariate analysis. Conclusion: According to the results of this study, female patients with a lower EBW who improve their postoperative eating behaviour have the best chance of success following LAGB.

\footnotetext{
* Both authors contributed equally to this work.
}

\section{Introduction}

Within 5 years, the number of operations for bariatric surgery has increased more than five-fold in most industrialised countries due to the worldwide epidemic of obesity [1]. In recent cohort studies it has been shown that bariatric surgery reduced long-term mortality in morbidly obese patients [2,3]. Thus, surgery is considered the treatment of choice for morbid obesity at the present time. Laparoscopic adjustable gastric banding (LAGB) as a purely restrictive procedure with a simple and standardised technique has been found to provide significant weight loss in morbid obesity and a good reduction in comorbidities with a very low mortality rate. However, the outcome of this procedure is variable and absence of weight loss or even weight regain may occur in the longterm postoperative period [4]. Additionally, LAGB-associated complications like port problems, band migration/leakages, and slippage leading to a reoperation rate of up to $8.5 \%$ have been recently reported $[5,6]$. It has been hypothesised that success or failure of LAGB is not only a function of the surgical procedure alone. Obviously, other criteria like physical activity, social and familiar circumstances as well as different personality traits with impact on eating behaviour and adjustment following surgery play a critical role in achieving and maintaining weight reduction [7]. Taking these considerations into account, a gastric band-specific patient selection seems crucial for the prediction of success or failure after LAGB.

The aim of this study was to examine individual pre- and postoperative criteria predicting the outcome of gastric banding.

\section{Patients and Methods}

Patients

A number of 85 patients were operated for morbid obesity with gastric banding between 1999 and 2005. The laparoscopic approach was standardised in all patients and has been described before [8]. The Swedish adjustable gastric banding (SAGB; Obtech, Ethicon Endo-Surgery, Norderstedt, Germany)

$\begin{array}{ll}\text { KARGER } & \text { ( 2009 S. Karger GmbH, Freiburg } \\ \text { Fax +497614520714 } & \text { Accessible online at: } \\ \begin{array}{l}\text { E-mail Information@Karger.de } \\ \text { www.karger.com }\end{array} & \text { www.karger.com/ofa }\end{array}$

Dr. med. Andreas Thalheimer

Zentrum Operative Medizin, Chirurgische Klink und Poliklinik

Universitätsklinikum Würzburg

Oberdürrbacher Straße 6, 97080 Würzburg, Germany

Tel. +499312 01-38714

Thalheimer_A@chirurgie.uni-wuerzburg.de 
was used in all cases and positioned through the pars flaccida technique. The patients were considered as candidates for LAGB when the following conditions were met: age $>18$ years and body mass index $(\mathrm{BMI})>40 \mathrm{~kg} / \mathrm{m}^{2}$ or $\mathrm{BMI}>35 \mathrm{~kg} / \mathrm{m}^{2}$ with obesity-related comorbidities. In total, there were 69 women and 16 men with a median age of 40 years (18-64 years). Preoperative medium body weight (BW) was $136 \mathrm{~kg}(88-202 \mathrm{~kg})$ with a BMI of $49 \mathrm{~kg} / \mathrm{m}^{2}\left(37-73 \mathrm{~kg} / \mathrm{m}^{2}\right)$ and an excess body weight (EBW) of $52 \mathrm{~kg}$ (17$110 \mathrm{~kg}$ ). A medium of 2.4 obesity-related comorbidities per patient was recorded.

\section{Study Design}

Primary endpoints of this study were, firstly, the rate of success after gastric banding defined as excess body weight loss (EBWL) $>50 \%$ without band removal and, secondly, the rate of failure after gastric banding defined as EBWL $<20 \%$ and/or band removal.

\section{Data Collection}

All patients were compared in terms of general features, comorbidities, postoperative complications, social and familiar circumstances, eating behaviour, and physical activity. Eating behaviour and eating attitudes were evaluated according to the following patterns: Sweet eating was diagnosed when the patient continuously craved simple carbohydrates triggered by emotional and physiological situations. Stress eating was diagnosed when any food ingestion other than carbohydrates could be triggered by emotional and physiological stress situations. Additionally, patients were asked regarding events of vomiting of food remnants after eating and considered positive if this happened more than once a day. The patients were considered as physically active when endurance sports (e.g. swimming, running, power walking, aerobics, cycling) was performed more than 3 times a week for more than $30 \mathrm{~min}$.

\section{Statistical Analysis}

All data are presented as median (range) unless otherwise stated. Statistical evaluations used Fisher's exact test, $\chi^{2}$ tests, and nonparametric tests (MannWhitney U test, Kruskal-Wallis test). Multivariate analysis was done using logistic regression with backward elimination of variables with SAS ${ }^{\circledR}$ software (SAS Institute, Cary, NC, USA). $\mathrm{P}<0.05$ was considered significant.

\section{Results}

11 of 85 patients were lost to follow-up (13\%) in a median of 27 months (8-90 months). 2 patients were excluded due to band infection as this complication was considered independent from postoperative compliance or misbehaviour. Another patient was excluded after band removal and switch to gastric bypass at the patient's request, leaving 71 patients $(84 \%)$ for the analysis in this study. The groups for statistical analyses in this study were success $(\mathrm{n}=26,37 \%)$ versus no success $(\mathrm{n}=45,63 \%)$ and failure $(\mathrm{n}=14$, $20 \%)$ versus no failure $(\mathrm{n}=57,80 \%)$.

\section{General Outcome}

General outcome of all patients is summarised in table 1. Overall, there was no mortality, with a morbidity rate of $21.9 \%$.

Procedure-related complications were band migration and port site infection in $2.4 \%$, band leakage and pouch dilatation in $3.7 \%$, port dislocation in $7.3 \%$, and band slippage in $8.5 \%$. Due to procedurerelated complications, $17.1 \%$ of all patients $(n=14)$ required one or more reoperations under general anaesthesia, including band replacement $(9.8 \%)$ and band removal $(4.9 \%)$.
Table 1. General outcome of LAGB in all patients

\begin{tabular}{lcl}
\hline & Median & Range \\
\hline Body weight $(\mathrm{kg})$ & 115 & 70 to 200 \\
BMI $\left(\mathrm{kg} / \mathrm{m}^{2}\right)$ & 38 & 24 to 66 \\
Excess weight $(\mathrm{kg})$ & 28 & -17 to 102 \\
EWL $(\mathrm{kg})$ & 23 & -30 to 100 \\
EBWL $(\%)$ & 43.2 & -41 to 171.5 \\
Loss of BMI $\left(\mathrm{kg} / \mathrm{m}^{2}\right)$ & 8.0 & -9 to 35 \\
\hline
\end{tabular}

Table 2. Relevant factors predicting success or failure of gastric banding

\begin{tabular}{llll}
\hline Criteria & RR (odds ratio) & $95 \%$ CI & p value \\
\hline Success vs. no success & & & \\
Female sex & 5.5 & $1.2-26.1$ & 0.023 \\
BMI < 50 kg/m ${ }^{2}$ & 3.0 & $1.2-7.7$ & 0.024 \\
Port dislocation & 3.3 & $0.37-29.3$ & 0.401 \\
Reoperation & 4.5 & $0.9-21.5$ & 0.068 \\
Band replacement & 1.9 & $0.4-10.2$ & 0.704 \\
Sweet eating & 0.2 & $0.05-0.7$ & 0.008 \\
Stress eating & 0.1 & $1.3-11.7$ & 0.015 \\
Physical activity, postop. & 4.2 & $1.5-11.7$ & 0.007 \\
\hline Failure vs. no failure & & & \\
Male sex & 3.9 & $1.2-12.7$ & 0.038 \\
BMI > 50 kg/m ${ }^{2}$ & 1.9 & $0.7-5.5$ & 0.291 \\
Port dislocation & 8.9 & $1.5-53.3$ & 0.019 \\
Reoperation & 5.2 & $1.5-17.7$ & 0.011 \\
Band replacement & 0.5 & $0.06-4.2$ & 0.678 \\
Sweet eating & 0.6 & $0.2-2.0$ & 0.530 \\
Stress eating & 0.4 & $0.1-1.2$ & 0.121 \\
Physical activity, postop. & 4.0 & $1.0-15.7$ & 0.045 \\
\hline CI Confidence interva & & & \\
\hline
\end{tabular}

$\mathrm{CI}=$ Confidence interval.

Regarding obesity-related comorbidities, there was a significant improvement in patients with a successful outcome following LAGB compared to patients with no success in hypertension $(p=0.002)$, diabetes $(p=0.07), \quad$ degenerative joint disease $(\mathrm{p}<0.001)$ and dyspnoea $(\mathrm{p}<0.001)$.

\section{Predicting Outcome}

All relevant factors predicting success or failure following gastric banding are listed in table 2 .

Female sex predicted success after LAGB whereas male sex was identified as a statistically significant predictor for failure. Furthermore, the baseline BMI was detected as an additional objective preoperative parameter predicting success or failure following LAGB with a cut-off value of $50 \mathrm{~kg} / \mathrm{m}^{2}$. Port- or gastric banding-related complications leading to a reoperation did not seem to be of predictive value regarding success following LAGB, whereas port dislocation and reoperations were predictors of failure. Changes in eating behaviour after LAGB, especially the termination of sweet and stress eating, were a significant predictor of success whereas this did not play a role in predicting a failure after LAGB. Improving the physical activity postoperatively was also a significant predictor of success as much as the absence of activity was a significant predictor of failure following LAGB. 
Baseline EBW and eating behaviour were identified as independent predictors of success in multivariate analysis $(p=0.008)$. In contrast, only EBW was of significant importance in a multivariate analysis when predicting failure $(\mathrm{p}=0.005)$.

\section{Discussion}

Although there is little doubt that gastric banding is an effective treatment for severe obesity, there is a large variability in weight outcome ranging from fairly good results to complete failures [9, 10]. Besides physiological and technical reasons contributing to failure after LAGB, motivational and psychological factors have been considered to have an important impact on the postoperative course following gastric banding [11]. This has been challenged by another report in which the role of personality assessments as psychological screening for admission has been considered as less relevant for the prediction of a poor or successful weight outcome after bariatric surgery [12]. In a study published recently, patients treated with LAGB had a lower short-term morbidity than those treated with Roux-en-Y gastric bypass, but reoperation rates were higher among patients who received LAGB [13]. Thus, valid factors for the prediction of success or failure following LAGB are mandatory.

In the present study, baseline BMI with a cut-off value of $50 \mathrm{~kg}$ / $\mathrm{m}^{2}$ was identified as a predictor of success or failure following LAGB which is in accordance with other studies [14, 15]. It is an accepted agreement that super-obese patients may require more complex procedures, e.g. Roux-en-Y gastric bypass, to achieve satisfactory weight loss [13]. Furthermore, gender seems to be an important factor when predicting success or failure: female sex was significantly associated with success while male sex predicted failure. Keeping in mind that the baseline EBW was the main predictor of success in the multivariate analysis, we can state that gender and preoperative weight are of major importance for the decision whether patients should be treated with LAGB or not. This is of high relevance insofar as gender and baseline weight are objective preoperative parameters which are irrevocable. In contrast, postoperative modification of eating behaviour which predicted statistically significant success even in the multivariate analysis of this study is difficult to assess preoperatively. As long as valid and objective tools for the assessment of the patients' compliance do not exist, the preoperative predictive value of aspects like modification of eating behaviour, physical activity, and motivation for changing one's lifestyle is limited. Nevertheless, these aspects are obviously of great importance for the outcome following LAGB. Thus, a sufficient motivational and psychological support in the postoperative course is necessary to influence postoperative eating behaviour and physical activity. Regular postoperative meetings with nutrition technicians and psychologists addressing these behaviour modifications are absolutely recommendable and important for maintaining postoperative weight loss after gastric banding.

Complications after LAGB resulting in the necessity of reoperation were statistically significant in predicting the failure of the procedure. This, however, can be related to the fact that band removals were considered as failures and required a reoperation. Differences became insignificant when band removals were excluded from the failure group. Nevertheless, all situations leading to reoperations, like band migration, port site infections, pouch dilatation, and band slippage, should be avoided by a subtle, standardised operation technique and a consistent postoperative surveillance.

In conclusion, it has been shown that LAGB provides sustained weight loss in a large proportion of obese patients. The efficiency of this procedure can be increased by a reasonable preoperative patient selection. Its lower efficacy in comparison to malabsorptive procedures must be balanced against its lower rate of life-threatening complications as well as the postoperative adjustability and its complete reversibility. Based on the present analysis, we recommend LAGB for female patients with a BMI $<50 \mathrm{~kg} / \mathrm{m}^{2}$ and an evident motivation to change eating behaviour and physical activity postoperatively. This is based partly on the personal impression of the surgeon who still has a significant influence on the final treatment decision. Gender and baseline BMI, however, are objective criteria which might help to reach the right decision.

\section{Disclosure}

The authors declared no conflict of interest. 


\section{References}

1 Santry HP, Gillen DL, Lauderdale DS: Trends in bariatric surgical procedures. JAMA 2005;294 1909-1917.

-2 Sjöström L, Narbro K, Sjöström CD, Karason K Larsson B, Wedel H, Lystig T, Sullivan M, Bouchard C, Carlsson B, Bengtsson C, Dahlgren S, Gummesson A, Jacobson P, Karlsson J, Lindroos AK, Lönroth H, Näslund I, Olbers T, Stenlöf K, Torgerson J, Agren G, Carlsson LM: Effects of bariatric surgery on mortality in Swedish obese subjects. N Engl J Med 2007;357:741-752.

-3 Adams TD, Gress RE, Smith SC, Halverson RC, Simper SC, Rosamond WD, Lamonte MJ, Stroup AM, Hunt SC: Long-term mortality after gastric bypass surgery. N Engl J Med 2007;357:753-761.

4 O'Brien PE, Brown WA, Smith A, McMurrick PJ, Stephens M: Prospective study of a laparoscopically placed, adjustable gastric band in the treatment of morbid obesity. Br J Surg 1999; 86:113-118.

$\checkmark 5$ Zehetner J, Holzinger F, Triaca H, Klaiber C: A 6-year experience with the Swedish adjustable gastric band. Prospective long-term audit of laparoscopic gastric banding. Surg Endosc 2005; 19:21-28.
Bueter M, Maroske J, Thalheimer A, Gasser M, Stingl T, Heimbucher J, Meyer D, Fuchs KH, Fein M: Short- and long-term results of laparoscopic gastric banding for morbid obesity. Langenbecks Arch Surg 2008;393:199-205.

7 van Hout GC, van Oudheusden I, van Heck GL: Psychological profile of the morbidly obese. Obes Surg 2004;14:579-588.

$>8$ Freys SM, Tigges H, Heimbucher J, Fuchs KH, Fein M, Thiede A: Quality of life following laparoscopic gastric banding in patients with morbid obesity. J Gastrointest Surg 2001;5:401-407.

9 Weiner R, Datz M, Wagner D, Bockhorn H: Quality-of-life outcome after laparoscopic adjustable gastric banding for morbid obesity. Obes Surg 1999;9:539-545.

10 Belachew M, Belva PH, Desaive C: Long-term results of laparoscopic adjustable gastric banding for the treatment of morbid obesity. Obes Surg 2002;12:564-568.

11 DeMaria EJ, Sugerman HJ, Meador JG, Doty JM, Kellum JM, Wolfe L, Szucs RA, Turner MA: High failure rate after laparoscopic adjustable silicone gastric banding for treatment of morbid obesity. Ann Surg 2001;233:809-818.
Larsen JK, Geenen R, Maas C, de Wit P, van Antwerpen T, Brand N, van Ramshorst B: Personality as a predictor of weight loss maintenance after surgery for morbid obesity. Obes Res 2004;12: 1828-1834.

3 Tice JA, Karliner L, Walsh J, Petersen AJ, Feldman MD: Gastric banding or bypass? A systematic review comparing the two most popular bariatric procedures. Am J Med 2008;121:885-893.

14 Forsell P, Hallerback B, Glise H, Hellers G: Complications following Swedish adjustable gastric banding: a long-term follow-up. Obes Surg 1999;9:11-16.

5 De Panfilis C, Cero S, Torre M, Salvatore P, Dall'Aglio E, Adorni A, Maggini C: Utility of the temperament and character inventory (TCI) in outcome prediction of laparoscopic adjustable gastric banding: preliminary report. Obes Surg 2006;16:842-847. 